\title{
FULGURACIÓN CON LÁSER DE LA RECIDIVA DE TUMORES VESICALES SUPERFICIALES BAJO ANESTESIA LOCAL. DISEÑO DE PROCESOS*
}

\author{
Drs. María Carmen Cano-García ${ }^{1,2}$, Tomás Fernández-Aparicio ${ }^{2}$, \\ Guillermo Hidalgo-Agulló ${ }^{2}$, Leandro Reina-Alcaina ${ }^{2}$, Carlos Carrillo-George ${ }^{2}$, \\ Ángela Rivero-Guerra ${ }^{2}$, Bernardino Miñana-López ${ }^{2}$

\footnotetext{
Servicio de Urología. Hospital General Universitario José Ma Morales Meseguer. Murcia.

España.
} \\ 2 Servicio de Urología. Hospital La Inmaculada. Huércal Overa. Almería.
}

\begin{abstract}
Laser fulguration of superficial bladder tumor recurrence under local anesthesia. Design process
\end{abstract}

Introduction: The process design is one of the most interesting tools to ensure the quality of health care before the start of an activity. Although the gold standard treatment of superficial bladder tumors remains transurethral resection (TUR), the onset of laser energy source with better endourological systems allows us to adopt alternative therapies. The aim of this pilot study is to describe the design and protocol in 37 patients with a novel process consisting of outpatient treatment under local anesthesia of bladder tumors with holmium laser. Material and Methods: Pilot study includes 37 patients between January 2012 and December 2013, for the development of a process of holmium laser bladder fulguration without anesthetic infiltration in outpatient study. It analyzes and studies the procedure tolerance, development of immediate complications, visual analog scale (VAS) of pain, patient satisfaction, hospital stays avoided, problems during the application process and development. Results: The mean age of the patients was $69.2 \pm 10.3$ years, $100 \%$ of patients prefer this procedure instead conventional transurethral resection and VAS rating presenting $\leq 3$. There were no important complications. Only one patient was admitted at hospital due to hematuria resolved without surgical treatment. Conclusions: The development and implementation of fulguration of superficial papillary bladder tumors with holmium laser process is simple, well tolerated, ambulatory and without complications, with no need of hospital stay.

Key words: Process Design, process Map, bladder neoplasia, superficial bladder cancer, holmium laser.

\section{Resumen}

Introducción: El diseño de procesos es una de las herramientas de mayor interés para asegurar la calidad de la asistencia sanitaria antes del comienzo de una determinada actividad. Aunque el gold standard del

\footnotetext{
No existe conflicto de intereses. No hay financiación.

Correspondencia: Dra. María del Carmen Cano-García mamen.cano@hotmail.com
}

* Recibido el 22 de julio de 2014 y aceptado para publicar el 17 de octubre de 2014. 
tratamiento de los tumores vesicales superficiales sigue siendo la resección transuretral (RTU), la aparición de la fuente de energía láser con mejores medios endourológicos nos permite adoptar otras alternativas terapéuticas. El objetivo de este estudio piloto es describir el diseño y protocolo en 37 pacientes de un proceso novedoso consistente en el tratamiento en régimen ambulatorio y bajo anestesia local intravesical de los tumores de vejiga con láser de holmium. Material y Métodos: Estudio piloto que incluye 37 pacientes entre enero de 2012 y diciembre de 2013, para la elaboración de un proceso de fulguración vesical con láser de holmium sin infiltración anestésica en régimen ambulatorio. Se analiza y estudia tolerancia al procedimiento, desarrollo de complicaciones inmediatas, escala visual analógica (EVA) del dolor, satisfacción del paciente, estancias hospitalarias evitadas, problemas durante la aplicación del proceso y desarrollo del mismo. Resultados: La edad media de los pacientes fue $69,2 \pm 10,3$ años, presentando puntuación EVA $\leq 3$. No existieron complicaciones importantes. Hubo un ingreso por hematuria tardía que se resolvió de forma conservadora. Conclusiones: La elaboración y aplicación del proceso de fulguración de tumores vesicales papilares superficiales con láser holmium es una técnica sencilla, con buena tolerancia, ambulatoria y sin complicaciones de interés, con eliminación de estancias hospitalarias.

Palabras clave: Diseño de procesos, mapa procesos, neoformación vesical, cáncer vesical superficial, láser de holmium.

\section{Introducción}

El gold standard en el tratamiento del cáncer vesical superficial de bajo grado sigue siendo la resección transuretral ${ }^{1}$, sin embargo, en los últimos años gracias al avance tecnológico y mejora en las fuentes de energía, la fulguración con láser de holmium ${ }^{2}$ se ha convertido en una alternativa al tratamiento convencional, fundamentalmente en las pequeñas recidivas papilares en casos de tumores $\mathrm{Ta}^{3}$. Además de la resección transuretral, otras opciones terapéuticas son posibles en el tratamiento del cáncer vesical superficial no músculo-infiltrante, como la vigilancia activa ${ }^{4}$ y el tratamiento mínimamente invasivo con láser ${ }^{5}$. La vigilancia activa es una opción terapéutica válida en pacientes con importantes comorbilidades, en tratamiento con anticoagulantes o con tumores menores de $5 \mathrm{~mm}^{4}$. El tratamiento ambulatorio mínimamente invasivo, fundamentalmente con láser, permite tratar a pacientes con tumores papilares de bajo grado menores a $5 \mathrm{~mm}$ y en número $\leq 5$, con unos resultados de seguridad y eficacia aceptables 5 .

La instauración de un nuevo procedimiento terapéutico en una unidad de urología requiere el diseño de un proceso, en este caso denominado "fulguración con láser holmium de la recidiva de cánceres vesicales superficiales de bajo grado, mediante anestesia local, sin necesidad de infiltración anestésica". La gestión por procesos es una forma de abordar la gestión de los recursos a través de todo el sistema sanitario, que incide directamente en la organización del trabajo. Tiene como objetivo aumentar su efectividad y eficiencia, pretendiendo generar servicios de salud accesibles, integrables, continuados, sustentados en la mejor evidencia clínica disponible, y orientados a la satisfacción de las necesidades y expectativas de los pacientes y los profesionales ${ }^{6,7}$.
La gestión por procesos es, además, uno de los criterios a analizar según el modelo EFQM (European Foundation for Quality Management) y cada vez tiene mayor importancia en la cultura asistencial de nuestro sistema sanitario, tal y como se describen en algunos trabajos ya publicados ${ }^{8,9}$. A través de la autoevaluación, el modelo EFQM pretende una gestión más eficaz y eficiente e identificar los puntos fuertes y débiles de la organización para el proceso de mejora continua ${ }^{10}$.

El objetivo de este estudio piloto es diseñar un proceso dentro de un área específica de la unidad de urología, analizando diferentes indicadores de calidad, para el tratamiento mediante fulguración con láser holmium de la recidiva de tumores vesicales papilares superficiales de bajo grado.

\section{Material y Métodos}

El estudio ha sido realizado entre enero de 2012 y diciembre de 2013, diseñando un grupo de trabajo multidisciplinar. La estrategia para el diseño del proceso fue, secuencialmente, la siguiente: reunión multidisciplinar, elaboración del mapa de procesos nivel 0 de la unidad, análisis de guías clínicas sobre tumores vesicales, elaboración de un grupo de trabajo, análisis de las actividades, responsables, circuito y flujo, identificación de "CASO" y requerimientos para la inclusión de los pacientes y pilotaje del proceso en 37 pacientes.

Se trata de un estudio piloto descriptivo en el que se incluyen 37 pacientes consecutivos con carcinoma vesical no músculo invasivo de bajo riesgo con recidiva $\leq 10 \mathrm{~mm}$ papilar y en número $\leq 5$ con citologías negativas y sin carcinoma in situ (CIS). Se excluyen del estudio pacientes con carcinoma vesical 
no músculo invasivo de riesgo intermedio o alto o con riesgo bajo con tumores mayores de $10 \mathrm{~mm}$ y de número mayor de 5 , sin que la presencia de otras comorbilidades fuese exclusión del estudio. Se realiza el procedimiento con anestesia local [Colocación de un gel lubricante estéril para catéteres (Cathejell®), con lidocaína. Se repite Cathejell ${ }^{\circledR}$ a los 5 min y se coloca sonda de nelaton e instilación de solución de $100 \mathrm{ml}$ de suero fisiológico con 2 ampollas de lidocaína al 2\% (400 mg/100 ml). Se retira la sonda y se esperan $10 \mathrm{~min}$ ], cistoscopio flexible y fibra de láser holmium 365 micras, aplicando una energía $0,5-0,8 \mathrm{~J}$ a $5 \mathrm{~Hz}$ para la fulguración tumoral (Figura 1 ), posterior instilación de mitomicina y procedimiento en régimen ambulatorio. Se elaboran mapas de procesos del área, así como diagramas de vías paralelas, útiles en la aplicación y puesta en marcha del proceso. Se analiza en este primer estudio piloto la tolerancia al procedimiento, desarrollo de complicaciones inmediatas, escala visual analógica (EVA), satisfacción del paciente (satisfecho o no satisfecho en relación al procedimiento de RTU convencional previo), clasificación del nivel de dificultad del procedimiento (baja, mediana, alta), problemas durante la aplicación del proceso y desarrollo del mismo.

El estudio fue aprobado por el Comité Ético del Hospital Morales Meseguer y los pacientes dieron su consentimiento por escrito para participar en el estudio.

\section{Resultados}

La dinámica y trabajo de grupo permitió dibujar el mapa 0 de procesos del Servicio de Urología en el año 2011, en el que queda reflejado todo el programa estratégico y plan de calidad del Servicio (Figura 2). La zona azul (representado en la Figura 2 como Hospital de Día-Azul) se corresponde con el área de procesos ambulatorios de la unidad de urología de nuestro hospital. Tras realizar un análisis estructurado de las actividades del proceso, quedó establecido: nombre y definición del proceso, responsable del proceso, límites del proceso, protocolos, actividades o tareas y responsables e indicadores y plan de monitorización, quedando reflejado en el diagrama de vías paralelas (Figura 3). No hubo ninguna incidencia en la implementación del proceso y el desarrollo del mismo en los pacientes en los que se aplicó y que cumplieron los criterios de inclusión en el estudio.

Los 37 pacientes sometidos a este procedimiento se completaron sin ninguna incidencia ni complicación inmediata, precisando tan sólo un caso de ingreso hospitalario por hematuria (no requirió transfusión, 3 días de ingreso hospitalario, 3 días de sondaje vesical), realizando todos los casos en régimen ambulatorio. Los resultados quedan reflejados en la Tabla 1, en la que destaca que la mayor parte de los pacientes fueron hombres con una edad media en torno a 70 años, con una tasa de complicaciones del $2,7 \%$. El $100 \%$ de los pacientes prefiere la fulguración con láser holmium en régimen ambulatorio respecto a la RTU convencional, presentando el $100 \%$ una puntuación en la EVA $\leq 3$ (Tabla 1) y el $100 \%$ se encuentra satisfecho con este procedimiento en lugar de la RTU convencional. No han existido problemas o incidencias de interés durante la aplicación del proceso y los profesionales que lo han llevado a cabo se encuentran satisfechos con el procedimiento (el $100 \%$ de los profesionales lo consideraron de dificultad técnica baja).

Como elemento añadido a los resultados ya comentados, es necesario reseñar que la dinámica de grupo creada generó la suficiente formación e inercia como para progresar en la línea estratégica de gestión por procesos, quedando asimismo dibujado el mapa 1 de procesos del área, denominada área o zona azul (Figura 4). En el mapa 1 se identificaron los subprocesos de administración, acogida, cistoscopia, programa de instilaciones, tratamiento ambulatorio de neoformaciones vesicales, maniobras endoscópicas y otros.

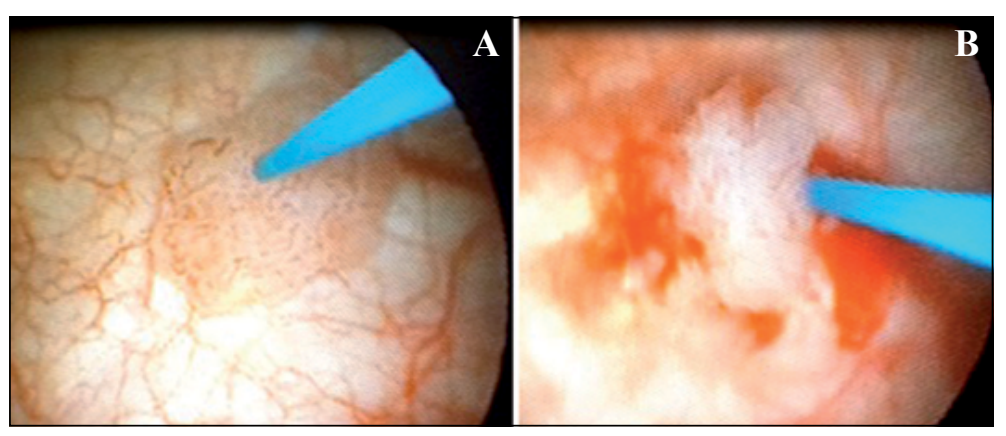

Figura 1. Imagen de cistoscopia de lesión papilar superficial de vejiga (A) $\mathrm{y}$ fulguración de la lesión con láser Holmium (B). 


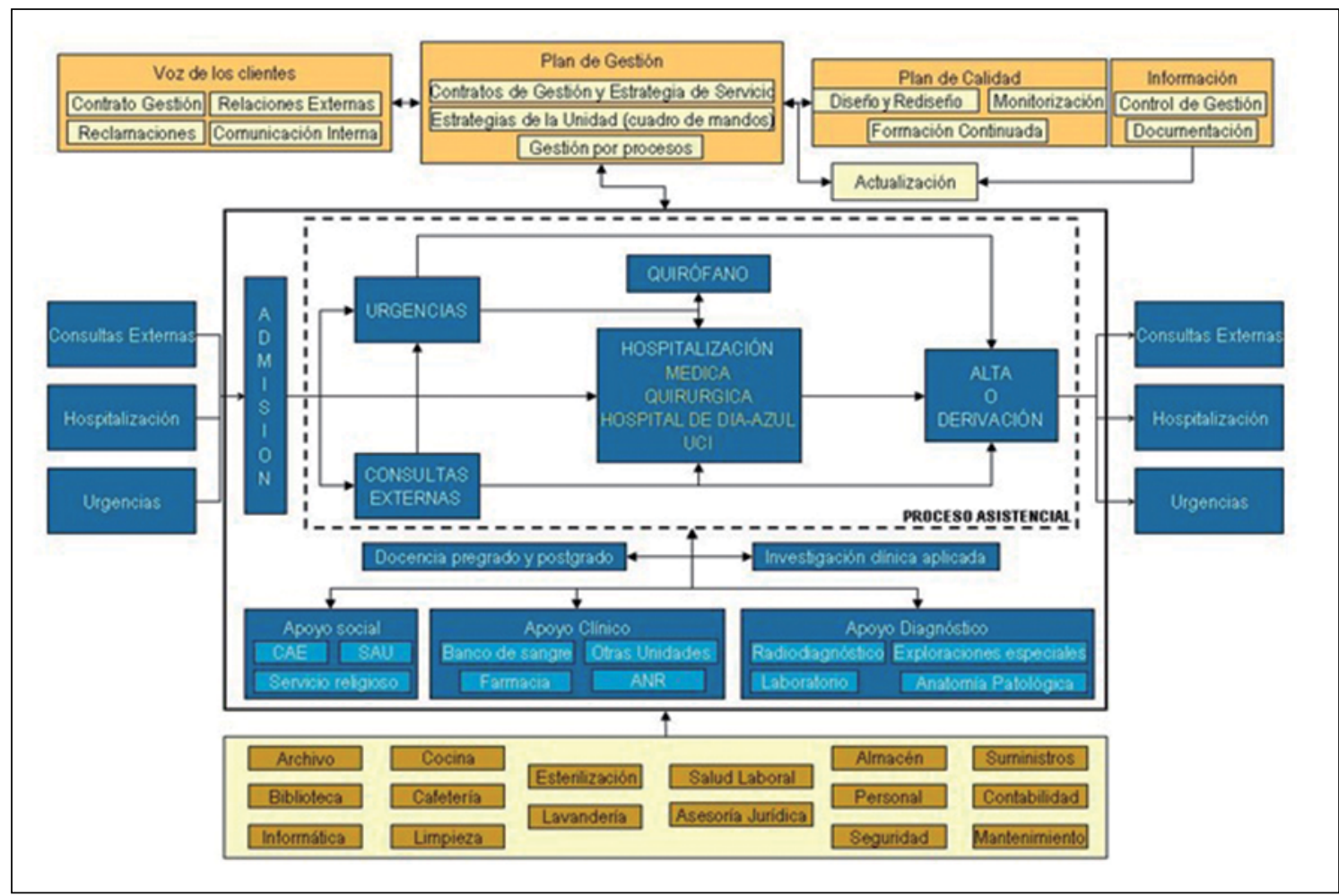

Figura 2. Mapa de procesos nivel 0 del Servicio de Urología del Hospital.

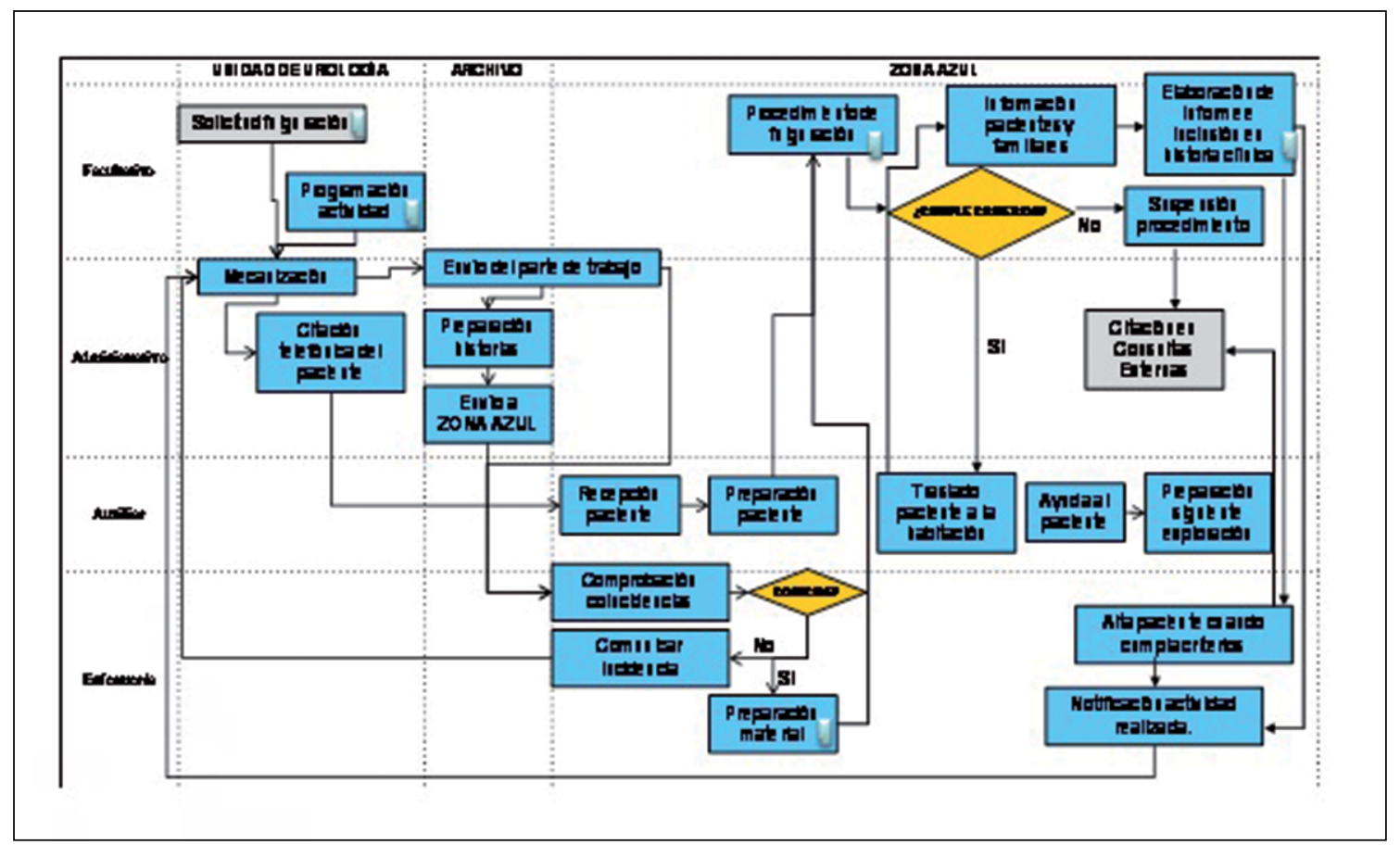

Figura 3. Diagrama de vías paralelas. 


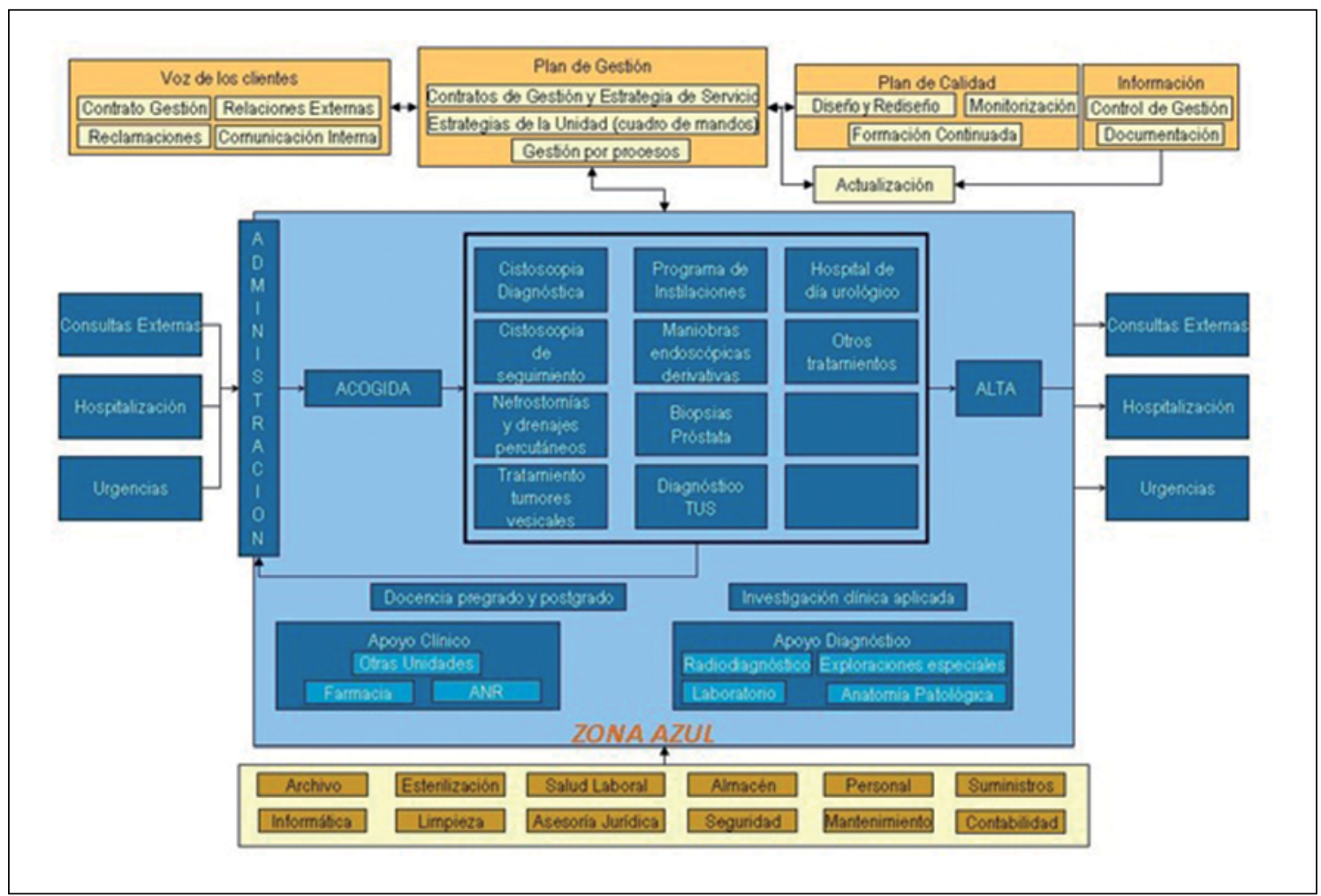

Figura 4. Mapa de procesos nivel 1 de la Zona Azul de Urología del Hospital.

Tabla 1. Resultados de los pacientes sometidos a fulguración vesical con láser holmium tras recidiva de tumor papilar superficial de bajo grado no músculo-infiltrante

\begin{tabular}{|lc|}
\hline $\mathrm{n}$ & $\begin{array}{c}\text { Pacientes sometidos } \\
\text { a fulguración vesical }\end{array}$ \\
\hline Edad (años) & 37 \\
Sexo (H/M) & $69,2 \pm 10,3$ \\
Tamaño tumoral (mm) & $83,8 \% \mathrm{H} / 16,2 \% \mathrm{M}$ \\
Complicaciones (\%) & $5,5 \pm 2,8$ \\
Puntuación EVA & 2,7 \\
& $0-48,6 \%$ \\
& $1-16,2 \%$ \\
& $2-21,6 \%$ \\
& $3-13,5 \%$ \\
\hline
\end{tabular}

\section{Discusión}

La gestión por procesos, es una forma de abordar la gestión de los recursos a través de todo el sistema sanitario, incidiendo de forma directa en la organi- zación del trabajo, teniendo como objetivo aumentar su efectividad y eficiencia, pretendiendo generar servicios de salud accesibles, integrables, continuados, sustentados en la mejor evidencia clínica disponible, $\mathrm{y}$ orientados a la satisfacción de las necesidades y expectativas de los pacientes y los profesionales, todo ello con la idea de aumentar la calidad en los sistemas de salud, identificando los puntos fuertes y débiles de la organización ${ }^{8,11}$.

Existen en la literatura diferentes estudios que tratan la recidiva del tumor vesical con láser holmium, habiendo demostrado que es un tratamiento coste-efectivo favorable especialmente en pacientes añosos y tumores de pequeño tamaño ${ }^{12,14}$. En los últimos años se ha realizado el procedimiento en tumores de mayor tamaño, habiéndose reflejado unos resultados satisfactorios y seguros para el paciente ${ }^{15}$. El procedimiento con mejor tasa coste-efectividad es la fulguración con láser de holmium sin instilación perioperatoria de mitomicina ${ }^{14}$, si bien, nuestro protocolo y proceso recoge la instilación de mitomicina para reducir el índice de recidivas y optimizar el procedimiento ambulatorio.

Aunque la experiencia es cada vez mayor en relación a la fulguración del tumor vesical con láser, 
es cierto, que no se han identificado trabajos que diseñen procesos y protocolos específicos de este procedimiento como el que hemos implementado ${ }^{16}$. Debido a que nuestros resultados se refieren sólo al pilotaje, podemos identificar las ventajas de este procedimiento respecto de la RTU convencional. Las ventajas más favorables de realizar este procedimiento son que se realiza en régimen ambulatorio, sin necesidad de anestesia general o loco-regional, con menor sangrado, con instrumento flexible menos doloroso para el paciente y sin necesidad de sondaje posterior; en comparación con los datos obtenidos en 2009 tras realización de 131 RTU vesicales de similares características ${ }^{17}$, con estancia media de 4,4 días (32 de ellos tuvieron complicaciones, con estancia media de 6,3 días), el ahorro de estancias estimado es de 162,8 días; sabiendo que el ingreso hospitalario del paciente con hematuria supuso 3 días, el ahorro de estancias fue 160 días. Son hechos importantes, teniendo en cuenta que el cáncer vesical es uno de los más frecuentes, afectando a más de 5 millones de personas en países desarrollados según se refleja en un estudio español reciente que analiza la prevalencia de la enfermedad ${ }^{18}$.

Como conclusión, el diseño de este proceso ha conseguido poner en marcha un conjunto de actividades secuenciales sin errores en su secuencia de aplicación y asegurando los criterios de calidad empleados. La experiencia adquirida con el trabajo de grupo ha permitido la formación del personal involucrado y avanzar en la estrategia de implementación de la gestión por procesos en una Unidad Clínica. Esto nos permitirá su aplicación y uso en la práctica clínica habitual y su comparación con otras alternativas terapéuticas.

\section{Agradecimientos}

A Miguel Ángel Arrabal Polo, Urólogo, AGS Norte de Almería. Hospital La Inmaculada. Huércal Overa (España).

\section{Referencias}

1. Babjuk M, Burger M, Zigeuner R, Shariat SF, van Rhijne BWG, Compérat E, et al. EAU Guidelines on NonMuscle-invasive Urothelial Carcinoma of the Bladder: Update 2013. Eur Urol. 2013;64:639-53.

2. Soler-Martínez J, Vozmediano-Chicharro R, MoralesJiménez P, Hernández-Alcaraz D, Vivas-Vargas E, Santos García-Vaquero I, et al. Holmium Laser Treatment for Low Grade, Low Stage, Noninvasive Bladder Cancer With Local Anesthesia and Early Instillation of Mitomycin C. J Urol. 2007;178:2337-9.

3. Herr HW, Donat SM, Reuter VE. Management of Low Grade Papillary Bladder Tumors. J Urol. 2007;178:1201-5.

4. Meeks JJ, Herr HW. Office-based Management of Nonmuscle Invasive Bladder Cancer. Urol Clin N Am. 2013;40:473-9.

5. O'Neil BB, Lowrance WT. Office-based Bladder Tumor Fulguration and Surveillance Indications and Techniques. Urol Clin N Am. 2013;40:175-82.

6. Saturno PJ. Seguridad del paciente. Marco conceptual. Enfoques y actividades. Manual del Máster en gestión de la calidad en los servicios de salud. Módulo 6: Seguridad del paciente. Unidad temática 33. $1^{\text {a }}$ Ed. Universidad de Murcia 2008. ISBN: 978-84-8371-750-9.

7. García Pascual A, de la Torre Burgoa A, Ruiz Pascual C, Torrego García JC, Silva Abuin JM, Velasco Fernández MC, y cols. Cáncer de vejiga. Guía para la gestión integrada de procesos asistenciales relacionados con el cáncer: "proyecto oncoguías". Ed. Junta de Castilla y León, 2003.

8. Fernández Fernández I, Fernández de la Mota E, Sanz Amores R. Gestión por procesos asistenciales: aplicación a un sistema sanitario público. Cuadernos de Gestión 2003;9:19-37.

9. Lorenzo S, Bacigalupe M, Arcelay A. Aplicación de la gestión por procesos a las unidades de atención al usuario. Rev Calidad Asistencial 2002;17:353-7.

10. Guía para la aplicación del Modelo EFQM de Excelencia en entidades de Acción Social. http://www.fundacionluisvives.org/upload/06/55/EFQM_def.pdf.

11. Ruiz López P, Martínez Hernández J, Alcalde Escribano J. Gestión de procesos en el Hospital Universitario 12 de Octubre. Rev Adm Sanit. 2006;4:233-49.

12. Wong KA, Zisengwe G, Athanasiou T, O’Brien T, Thomas K. Outpatient laser ablation of non-muscle invasive bladder cancer: is it safe, tolerable and cost-effective? BJU Int. 2013;112:561-7.

13. Donat SM, North A, Dalbagni G, Herr HW. Efficacy of office fulguration for recurrent low grade papillary bladder tumors less than $0.5 \mathrm{~cm}$. J Urol. 2004;171:6369.

14. Green DA, Rink M, Cha EK, Xylinas E, Chughtai B, Scherr DS, et al. Cost-effective treatment of low-risk carcinoma not invading bladder muscle. BJU Int. 2013;111:E78-84.

15. Kramer MW, Wolters M, Cash H, Jutzi S, Imkamp F, Kuczyk MA, et al. Current evidence of transurethral Ho: YAG and Tm: YAG treatment of bladder cancer: update 2014. World J Urol. 2014. doi 10.1007/s00345014-1337-y.

16. Cano García MC, Fernández Aparicio T, Alonso Frías MA, Jiménez Moreno R, Pietricicá BN, Miñana López B. Rediseño de procesos en un área intervencionista ambulatoria de Urología. XXIX Congreso de la Sociedad Española de Calidad Asistencial y VIII Congreso Regional de Calidad Asistencial, octubre de 2011.

17. Montoya-Chinchilla R, Miñana-López B, Pietricica B, 
M. C. CANO-GARCÍA y cols.

Cano García MC, Hidalgo Agulló G, Reina Alcaina L, y cols. Valoración del tratamiento de tumores uroteliales vesicales no músculo infiltrantes. Herramienta de ayuda para tratamiento basada en guías de práctica clínica europeas 2011. XXII Jornadas de la Asociación Murciana de Urología. 2011. Murcia.

18. Bernal-Pérez M, Souza DL, Romero-Fernández FJ, Gómez-Bernal G, Gómez-Bernal FJ. Estimation of bladder cancer projections in Spain. Actas Urol Esp. 2013;37:286-91. 